\title{
The Insertable B-Layer of the AtLas Experiment for the Run-2 at the Large Hadron Collider
}

\author{
Francesco Guescini ${ }^{\mathrm{a}}$, on behalf of the AtLas Collaboration \\ ${ }^{a}$ Université de Genève, DPNC, Quai Ernest-Ansermet 24, 1211 Genève 4, Switzerland \\ Francesco.Guescini@cern.ch
}

\begin{abstract}
The Pixel Detector of the ATLAs experiment has shown excellent performance during the whole Run- 1 of LHc. Taking advantage of the long shutdown, the detector was extracted from the experiment and brought to surface, to equip it with new Service Quarter Panels, to repair modules and to ease installation of the Insertable B-Layer (IBL). IвL is a fourth layer of pixel detectors and has been installed in May 2014 between the existing Pixel Detector and a new smaller radius beam pipe at a radial distance of $3.3 \mathrm{~cm}$ from the beam axis. The realization of the IвL required the development of several new technologies and solutions in order to overcome the challenges introduced by the extreme environment and working conditions, such as the high radiation levels, the high pixel occupancy and the need of an exceptionally low material budget. Two silicon sensor technologies have been adopted for the IBL modules: planar and 3D. Both of these are connected via bump-bonding to the new FE-I4 front-end read-out chip. Furthermore, the physics performance will be improved through the reduction of pixel size while, targeting for a low material budget, a new mechanical support using lightweight staves and a $\mathrm{CO}_{2}$ based cooling system have been adopted. An overview of the IвL project as well as the experience in its construction is presented, focusing on adopted technologies, modules and staves production, qualification of assembly procedure, integration of staves around the beam pipe and commissioning of the detector.
\end{abstract}

Keywords: Lhc, Atras, Pixel, IвL

\section{Introduction}

During the first shutdown of LHC [1], the AtLas [2] detector ${ }_{28}$ underwent some upgrades of the Pixel Detector [3] in prepa- ${ }_{29}$ ration for the Run-2 of LHc, which will take place between ${ }_{30}$ the years 2015 and 2017. The whole Atlas Run-1 Pixel De- ${ }_{31}$ tector has been extracted and brought to surface, where it has ${ }_{32}$ been equipped with new Service Quarter Panels and to allow ${ }_{33}$ for repair of broken modules. The Layer-2 DAQ hardware was ${ }_{34}$ also upgraded to withstand the data rates expected in Run-2. ${ }_{35}$ Moreover, the Diamond Beam Monitor was installed to perform ${ }_{36}$ bunch-by-bunch beam luminosity measurements [4]. Nevertheless, the main upgrade of the AtLas Pixel Detector for Run-2 consists of a new inner pixel layer, the Insertable B-Layer (IBL), ${ }^{37}$ which is positioned between the former inner layer of the Pixel Detector (B-Layer) and a new, smaller, beam pipe, at a radial distance of $3.3 \mathrm{~cm}$ from the beam axis. Two silicon sensor technologies have been employed for the construction of the IBL modules: planar and 3D [5]. Sensors are connected with bump-bonds to a new generation 130 nm Iвм Смоs Fe-I4 frontend read-out chip [6].

\section{2. Towards Run-2}

\section{${ }_{30}$ exp}

\author{
mas
} order of $10^{34} \mathrm{~cm}^{-2} \mathrm{~s}^{-1}$ and the bunch spacing will be reduced
to $25 \mathrm{~ns}$, therefore leading to stronger out-of-time pile-up conditions, higher occupancy levels and more shared hits. From a performance point of view, this will lead to worse tracking, vertex reconstruction and flavor tagging [7][8].

\section{The Insertable B-Layer}

The Insertable B-Layer, IBL, is the $4^{\text {th }}$ and innermost layer of the new Atras Pixel Detector (see figure 1). It has been designed for the purpose of improving tracking, vertexing and flavor tagging, as well as providing tracking redundancy in case of failures of the Layer 0 , the so called B-Layer, and compensating for inefficiencies due to radiation damage and module failures.

\subsection{Staves}

The IBL has a modular structure composed of 14 units, the staves, symmetrically arranged around the beam pipe with an overlap in $\phi$ (see figure 2). The staves have a support structure made of carbon fiber laminate and carbon foam, with a titanium cooling pipe inside. The total length, including services, 


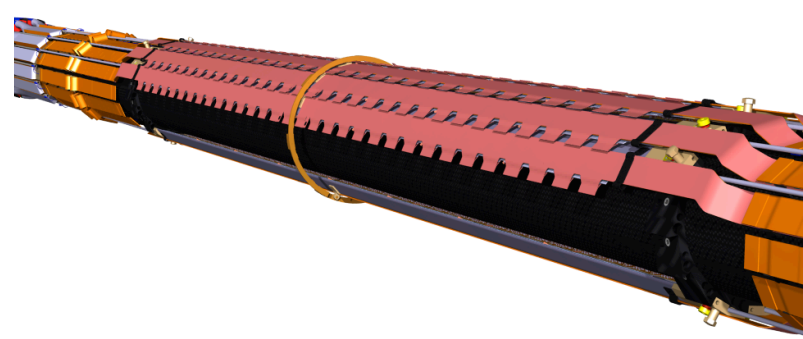

Figure 1: Schematic representation of the IBL showing its modular structure. Some of the staves are not represented.

is $7 \mathrm{~m}$ but only the central $66.4 \mathrm{~cm}$ are instrumented. Due to the 89 mechanical constraints given by the B-Layer, the IBL is located 90 in the region between $31 \mathrm{~mm}$ and $40 \mathrm{~mm}$ from the beam axis. ${ }^{91}$ The installation of the IвL inside the Pixel Detector was possible ${ }^{92}$ thanks to the replacement of the beam pipe used in Run-1 with ${ }^{93}$ a smaller one. The extremely light design structure of the IBL allowed its radiation length to be $1.9 \% \mathrm{X}_{0}$ including the sup- ${ }^{94}$ ports. Each stave is equipped with 20 pixel sensors: 12 double ${ }^{95}$ chip (planar) and 8 single chip (3D), for a total of 32 front-end ${ }^{96}$ chips per stave (see figure 2). The cooling of the staves is per- ${ }^{97}$ formed using two-phase $\mathrm{CO}_{2}$, with temperatures that can be as ${ }^{98}$ low as $-40^{\circ} \mathrm{C}$ in the cooling pipe and $-15^{\circ} \mathrm{C}$ on the sensors.

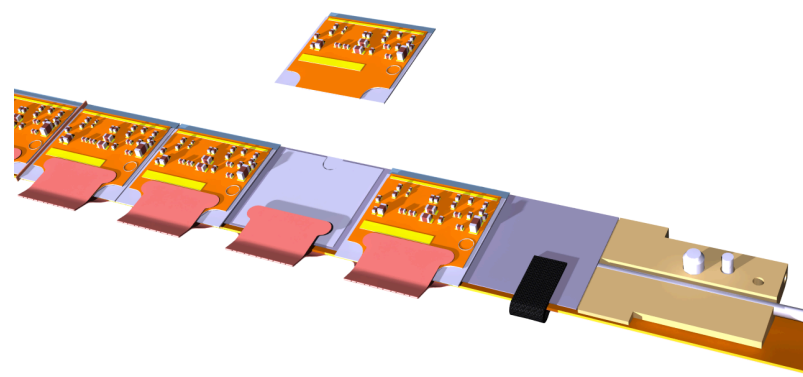

Figure 2: Schematic representation of one end of an IBL stave with the detail of 113 a 3D module.

\subsection{Modules}

IBL modules have $50 \times 250 \mu \mathrm{m}^{2}$ pixels, smaller than the ${ }^{118}$ $50 \times 400 \mu \mathrm{m}^{2}$ pixels in the other Pixel Detector layers. Two different silicon sensor technologies have been employed for the construction of the IBL modules: planar and 3D [5].

Planar sensors. Planar technology is well known and was al- ${ }_{121}$ ready used for the construction of the Run-1 Atras Pixel De- ${ }_{122}$ tector. IBL planar modules use $\mathrm{n}^{+}$-in-n sensors and have slim edges, in order to reduce the geometrical inefficiency. Pixels are $250 \times 50 \mu \mathrm{m}^{2}$ in size but the outermost pixels are twice as large, $500 \times 50 \mu \mathrm{m}^{2}$, and partially overlap with the guard rings. The sensors measure $4.132 \times 1.859 \mathrm{~cm}^{2}$ and are $200 \mu \mathrm{m}$ thick. Planar modules are double chip, meaning that they are composed of a single silicon sensor with $160 \times 336$ pixels read by two Fe-I4 front-end chips. Double chip modules are located in the central region of the staves. Sensors were produced by $\mathrm{CiS}^{1}$.

$3 D$ sensors. The 3D silicon technology has been adopted for the first time in a Lнс experiment for the IвL. Due to the processes involved in the production, the decision was taken to have only single chip 3D modules, i. e. sensors with $80 \times 336$ pixels read by one FE-I4 front-end chip. The sensors are $\mathrm{n}^{+}$in-p, measure $2.045 \times 1.875 \mathrm{~cm}^{2}$ and are $230 \mu \mathrm{m}$ thick. The use of 3D technology brings the advantage of having shorter collection distances and using lower depletion voltages but on the other hand gives a higher capacitance, hence larger noise values. Due to their better performance when hit by particles with a large incident angle, 3D modules have been placed in the forward regions, at the extremities of the staves, divided in two groups of four. 3D sensors have been produced by $\mathrm{CNM}^{2}$ and $\mathrm{FBK}^{3}$.

Front-end chip. A new front-end read-out chip was developed specifically for the IвL modules: the FE-I4, which replaces the older Fe-13 used for the Run-1 Pixel Detector modules. The Fe-I4 uses $130 \mathrm{~nm}$ Івм Cмоs technology. It is designed for $250 \times 50 \mu \mathrm{m}^{2}$ pixels and it has $80 \times 336$ channels and $8.7 \cdot 10^{7}$ transistors. It is $20.2 \times 18.8 \mathrm{~mm}^{2}$ large and $150 \mu \mathrm{m}$ thick. Due to the large fluences it will have to face at $33 \mathrm{~mm}$ from the interaction point, the FE-I4 is designed to withstand a flux of $5 \cdot 10^{15} 1 \mathrm{MeV}$ equivalent neutrons per $\mathrm{cm}^{2}$. The front-end is connected to the sensors with bump-bonds.

Almost 700 modules have been produced between January and October 2013, with a yield of $75 \%$ for the double chips and $62 \%$ for the 3D. Lower yield values were obtained in the first production batch due to a problem in the bump-bonding process, which caused open bumps and merged pixels. The issue was solved by performing a flux-free bump-bonding procedure. Optical inspections and electrical tests have been done on the modules at the production sites and at the stave loading site. A comprehensive list of tests was performed to ensure the quality, including I-V tests, analog and digital signal injection tests, threshold and noise tests. Modules have been subsequently ranked based on the number of bad pixels equivalents. Only those with less than $1 \%$ pixel defects per front-end have been loaded onto the staves.

\section{Stave loading}

After the selection of the best modules available, these were fixed onto the staves using two glue drops per front-end; a layer of thermal grease was deposited on the remaining surface to

\footnotetext{
${ }^{1} \mathrm{CiS}$ : Forschungsinstitut fur Mikrosensorik und Photovoltaik GmbH, Konrad-Zuse-Strasse 14, 99099 Erfurt, Germany.

${ }^{2}$ CNM: Centro Nacional de Microelectronica (CNM-IMB-CSIC), Campus Universidad Autonoma de Barcelona, 08193 Bellaterra (Barcelona), Spain.

${ }^{3}$ FBK: Fondazione Bruno Kessler (FBK), Via Sommarive 18, 38123 Povo di Trento, Italy.
} 
ensure good thermal conductivity. Flexible connectors, called ${ }_{143}$ wings, for clock, data and power were then glued on the modules and the two wire-bonded together. 20 staves have been ${ }^{144}$ produced in a one year long effort (see figure 3). A total of $428^{145}$ modules has been loaded, out of which 28 are the modules re- ${ }^{146}$ placed after loading (see figure 4). Most of these 28 modules $^{147}$ were replaced due to accidents which happened during load- ${ }^{148}$ ing and re-wire-bonding, very few because of front-end failures ${ }^{149}$ and just 2 because of failed quality assurance. Staves have been ${ }^{150}$ optically and electrically inspected after loading.

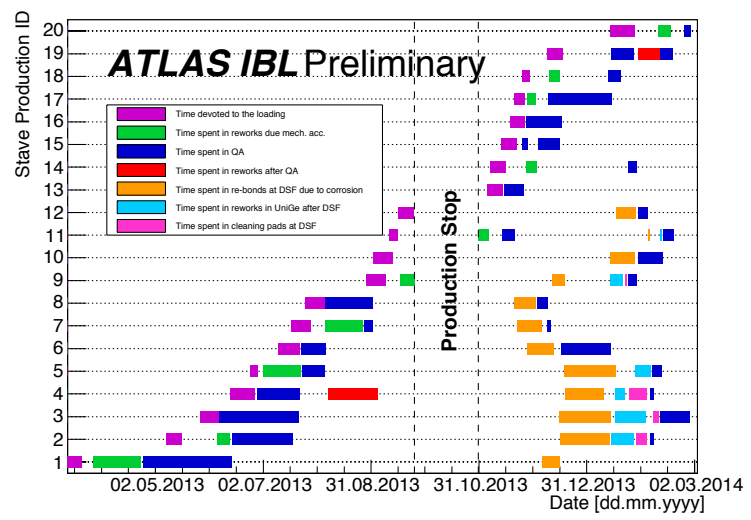

Figure 3: stave loading workflow [9].

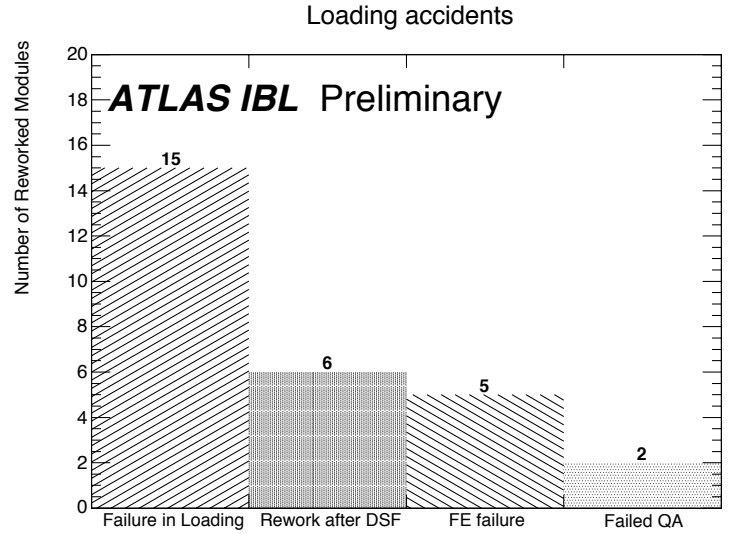

Figure 4: modules replaced divided by category [9]. 15 modules have been replaced due to failures during the stave loading procedure, 6 modules have been replaced after cleaning and re-wire-bonding at CERN Detector Silicon Facility, 5 because of front-end failures and 2 because of failed quality assurance.

Between September and October 2013, halfway through the process, the stave production was halted due to the observation of corroded wire-bonds and corrosion residuals (see figure 3). An investigation of the phenomenon revealed the presence of $\mathrm{Cl}$ and $\mathrm{F}$ associated with the corrosion products and the origin ${ }_{158}$ of the problem was traced back to the staves being exposed to ${ }_{159}$ humidity. Half of the staves was then cleaned and wire-bonds ${ }_{160}$ re-done. The options of potting or coating wire-bonds with ure-161 thane was investigated but in the end the decision was taken not ${ }_{162}$ to apply either of these.

\section{Stave quality assurance}

Extensive tests of the staves have been performed to ensure the quality and identify the best 14 to be loaded [10]. Optical inspections, electrical tests, tuning and calibration of the modules have been performed.

Figure 5 shows the results of the threshold measurements performed on the modules of the 14 IBL staves after tuning for a threshold of $1500 \mathrm{e}^{-}$and 10 ToT (1 ToT, Time over Threshold, corresponds to $25 \mathrm{~ns}$ ) for a $16000 \mathrm{e}^{-}$signal at $-12^{\circ} \mathrm{C}$. The values obtained are well aligned along the desired threshold value.

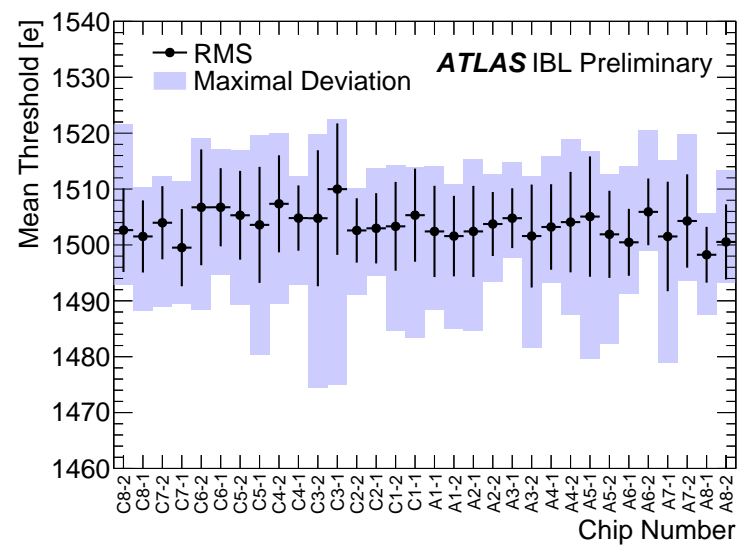

Figure 5: threshold scan results for the modules of the 14 IBL staves [10]. The $\mathrm{x}$ axis indicates the position of the front-end along the stave.

Figure 6 shows the results of the noise tests performed on the 14 IBL staves after tuning. The results are divided in normal and long pixels for the planar modules and $\mathrm{CNM}_{\mathrm{NM}}$ and $\mathrm{F}_{\mathrm{BK}}$ 3D modules. It is possible to observe that $3 \mathrm{D}$ modules have slightly larger noise values due to their design geometry.

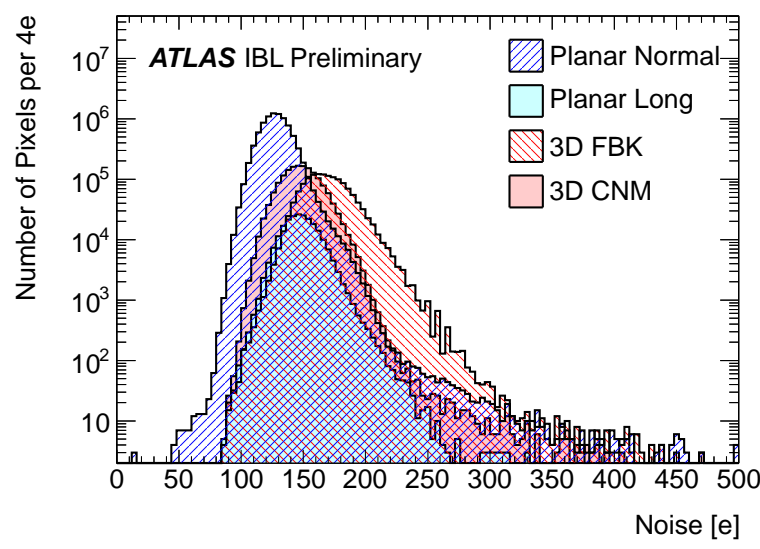

Figure 6: noise scan results for the modules of the 14 IBL staves [10].

Source scans have been performed using a ${ }^{90} \mathrm{Sr}$ source to determine disconnected and merged bumps. Staves have been thermal cycled to investigate possible cases of fast aging and infant mortality. Staves were ranked based on the equivalent number of bad pixels. Each of the 14 best staves selected for the IBL integration has less than $0.2 \%$ equivalent bad pixels. 


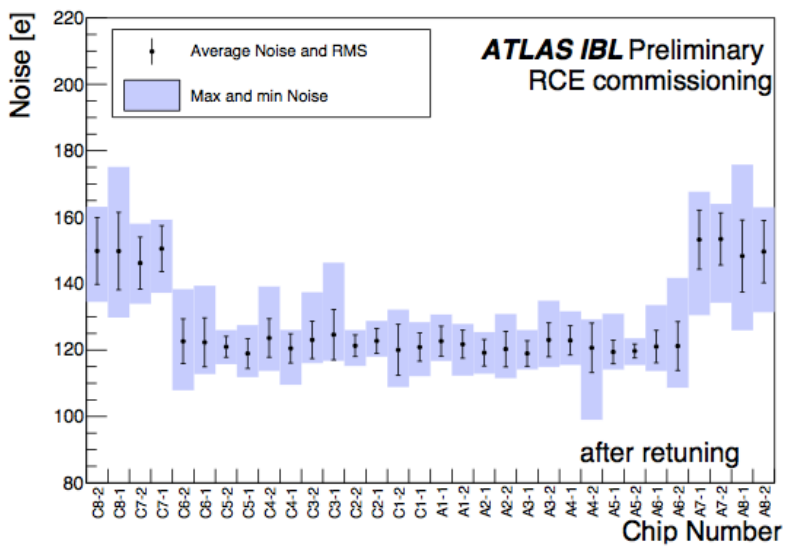

Figure 7: noise scan results for the modules of the 14 IBL staves after IBL insertion [9].

\section{Stave integration}

Staves have been integrated on the IвL Positioning Tube one by one and then electrically tested. The adjacent staves have also been tested in order to ensure that no damage had occurred during the procedure. The integration order was chosen to have, as much as possible, uniform performance of the IвL in the $\eta-\phi$ space and to avoid any possible concentration of bad pixels in a localized region. The whole procedure took almost two months and all of the modules were working. The final electrical tests performed on the assembled IBL confirmed the results obtained during quality assurance.

\section{Installation and commissioning}

IBL was lowered in the AtLAs cavern on May $5^{\text {th }}, 2014$ and successfully inserted in the pre-existent Pixel Detector two days later. The performance of the IBL was thereafter tested showing that all of the modules were working after the insertion.

Figure 7 shows the results of the noise scan. As already observed in figure $6,3 \mathrm{D}$ modules, located at the edges of the ${ }_{197}$ staves, have slightly larger noise values.

The difference between the results of the noise tests performed during the quality assurance and those obtained during ${ }_{215}^{214}$ the commissioning in the pit show good consistency of the two216 (see figure 8). The large differences observed for the 3D mod- ${ }^{217}$ ules located at the right end of the staves (A side) were caused ${ }_{219}^{218}$ by a noisy power line in the quality assurance system and are $_{220}$ thus not imputable to malfunctions of the modules.

\section{Expected performances}

Preliminary studies on the expected performances of the ${ }_{227}^{226}$ AtLas detector with the IBL are presented in [7]. The vertex 228 reconstruction capabilities and the resolution on the impact pa-229 rameters of tracks are expected to be significantly improved, es- ${ }^{230}$ pecially for particles at low $p_{\mathrm{T}}$. A notable improvement will be $\mathrm{e}_{232}^{231}$ brought to the flavor tagging, with the light-jet rejection almost 233

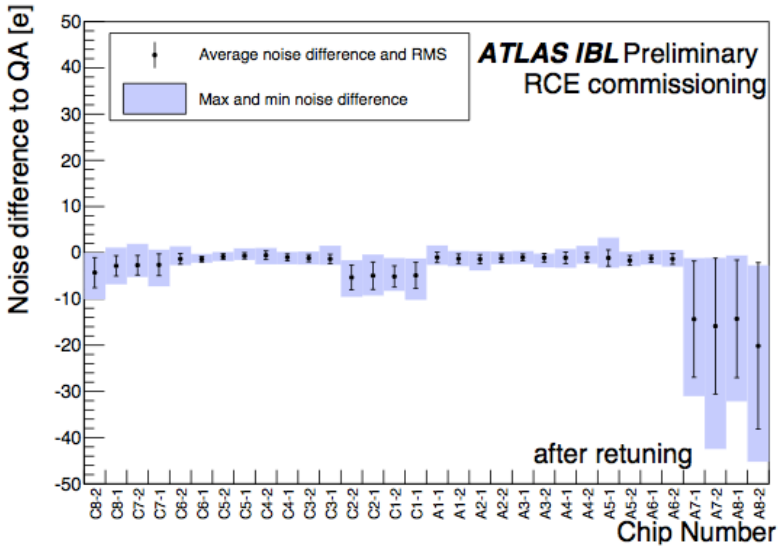

Figure 8: difference between the noise scan results for the modules of the 14 IBL staves performed during quality assurance and those performed after IBL insertion [9]. The large differences observed for the last four modules on the A side are due to a noisy power line in the stave quality assurance system. All other differences are within acceptance.

doubled. The redundancy provided by the IBL on the B-Layer hits is expected to increment and exceed the b-tagging performance of AtLas even in a scenario where $10 \%$ of the B-Layer is lost due to failures.

\section{Conclusions}

In order to cope with the increased pile-up conditions, larger occupancy and worse tracking conditions expected for Run-2, the ATLAs detector has been upgraded, during the first LHC long shutdown, with a fourth, inner silicon pixel layer: the Insertable B-Layer, IBL. For the realization of the IBL, several new technologies had to be specifically developed. The tests performed during the different stages of the IBL production show encouraging results. Preliminary studies on the AtLas Pixel Detector for Run-2 show that the IBL can effectively be a valid response to the demanding conditions expected and reliably compensate for possible failures of the B-Layer.

\section{References}

[1] L. Evans and P. Bryant (editors), LHC machine, 2008, Journal of Instrumentation 3 S08001

[2] Atlas Collaboration, The Atlas Experiment at the Cern Large Hadron Collider, 2008, Journal of Instrumentation 3 S08003.

[3] G. Aad et al., Atras Pixel Detector electronics and sensors, 2008, Journal of Instrumentation 3 P07007.

[4] L. Jeanty on behalf of the AtLas Collaboration, Operational Experience with the AtLas Pixel Detector, Resmdd14, in press in the same conference proceeding.

[5] Atlas Ibl Collaboration, Prototype Atlas Ibl modules using the FEI4A front-end readout chip, 2012, Journal of Instrumentation 7 P11010, arXiv:1209.1906.

[6] V. Zivkovic at al., The FE-I4 pixel readout system-on-chip resubmission for the insertable B-Layer project, 2012, Journal of Instrumentation 7 C02050.

[7] Atras Ibl Collaboration, Atras Insertable B-Layer Technical Design Report, 2010, CERN, CERN-LHCC-2010-013, ATLAS-TDR-19.

[8] Atlas Collaboration, Atras Insertable B-Layer Technical Design Report Addendum, 2012, CERN, CERN-LHCC-2012-009, ATLAS-TDR-19ADD-1. 
[9] Atras Collaboration, compilation of approved Pixel Detector plots, https://twiki.cern.ch/twiki/bin/view/AtlasPublic/ ApprovedPlotsPixel.

[10] Atras Collaboration, Atras Pixel IвL: Stave Quality Assurance, 2014, CERN, ATL-INDET-PUB-2014-006. 